\title{
Magnetic-field-induced bending and straining of Ni-Mn-Ga single crystal beams with high aspect ratios
}

\author{
Nikole J. Kucza ${ }^{\text {a }}$, Charles L. Patrick ${ }^{a}$, David C. Dunand ${ }^{b}$, Peter Müllner ${ }^{a^{*}}$ \\ ${ }^{a}$ Department of Materials Science \& Engineering, Boise State University \\ Boise, ID 83725, USA \\ ${ }^{\mathrm{b}}$ Department of Materials Science \& Engineering, Northwestern University \\ Evanston, IL 60208, USA \\ * Corresponding Author: petermullner@boisestate.edu \\ Phone: 1.208 .426 .5136
}

Small monocrystalline beams of the magnetic shape-memory alloy (MSMA) Ni-Mn-Ga, with a square $1 \times 1 \mathrm{~mm}^{2}$ cross section and length between 2 and $10 \mathrm{~mm}$, with the $10 \mathrm{M}$ martensitic structure and all faces parallel to $\{100\}$, were subjected to rotating magnetic fields while being held at one end. The beams deform by both magnetic-field-induced straining (MFIS) and magnetic-torque-induced bending (MTIB), in directions parallel and perpendicular to the beams longitudinal axis, respectively. With the field parallel to the beam axis, the beams were straight and short. Upon field rotation, the beam elongated and bent in the direction of the field. When the field reached $90^{\circ}$, the beam deflected rapidly and took a bent shape oriented in the opposite direction. Upon further field rotation, bending strain and axial strain decreased until the beam was short and straight again with the field at $180^{\circ}$. With increasing beam aspect ratio, the bending component increases while the total axial strain remains constant. MTIB - a natural but so far 
neglected response of long MSMA samples exposed to a transversal magnetic field occurs during switching of current linear (one-dimensional) actuators, thus causing friction losses, wear, and fatigue. However, MTIB provides the opportunity to actuate high aspect ratio MSMA continuously and smoothly in all directions (in three dimensions), thus mimicking slender biological actuating structures such as microorganism flagella tails and fins of fish, heart valves, leaves and petals of plants, and wings of birds or insects.

Keywords: Magnetic shape memory alloys; $\mathrm{Ni}_{2} \mathrm{MnGa}$; Deformation twinning; Magnetic properties; Magneto-mechanics

\subsection{Introduction}

Several designs for linear actuators, utilizing novel magnetic shape memory alloys (MSMA), have been proposed and prototyped $[1,2,3,4,5,6]$. These designs rely on single crystal samples of near-stoichiometric $\mathrm{Ni}_{2} \mathrm{MnGa}$ with a pseudo-tetragonal (10M) structure, which show low twinning stress, fast actuation response, and large twinning strain of $6 \%[7,8,9]$. Researchers have shown that $\mathrm{Ni}_{2} \mathrm{MnGa}$ samples with a comparatively large length to width aspect ratio require guides and tend to bend in addition to elongation $[10,11]$. Both effects lead to friction and may reduce the lifetime of actuators. $\mathrm{Ni}_{2} \mathrm{MnGa}$ single crystals are anisotropic and to achieve maximum output work the shape and the local magnetic structure need to be considered [11]. Several research groups used computer models to predict the spatially resolved deformation in three dimensions, to aid in the design of actuator devices $[12,13]$. One of these models 
defines movement for a beam, fixed at one end and freestanding on the other end, bending due to a localized magnetic field applied at the free end of the beam [13]. A 2D coupled magneto-mechanical structural analysis algorithm was used to simulate bending achieved by applying a magnetic field to a small volume $\left(1 \times 1 \times 2.5 \mathrm{~mm}^{3}\right)$ at the tip of the freestanding beam, $20 \mathrm{~mm}$ in length. Chen et al. concluded that stress-induced martensite reorientation was the actuation mechanism for magnetic field-controlled bending in magnetic shape memory alloys (MSMA).

In a recent study, Zheng et al. [14] showed that oligocrystalline Ni-Mn-Ga wires with diameters of $\sim 0.3 \mathrm{~mm}$ and aspect ratios ranging from 12 to 56 bend in a magnetic field, due to a magnetic torque, just like iron and other ferromagnetic metal wires. Unlike iron which usually remains elastic, Ni-Mn-Ga showed plastic bending as a result of motion of twin boundaries due to stresses from the magnetic torque. This mechanism, dubbed magnetic-torque-induced bending (MTIB), is enabled by twinning and allows for a large number of reversible bending without the strain hardening and damage accumulation occurring in metals hinging or bending by dislocation-enabled plasticity.

Thus, long MSMA transducers naturally respond to magnetic fields by bending, resulting in a deflection rather than an elongation. This deflection must be suppressed in linear actuators. Most literature of magnetic-field-induced deformation addresses linear, uniaxial magnetic-field-induced strain (MFIS). In contrast, Ganor et al. [15] and Kanner et al. [16] proposed propulsion mechanisms for remotely activated micro-robots in water and fluids. They suggested mechanisms based on shearing and kinking associated with twinning. These mechanisms utilize shear that causes similar out-of-axis motion as bending rather than suppressing it as is required in linear actuators. Measured transient 
thrusts with a 10M Ni-Mn-Ga single crystal rod with aspect ratio 5 were much higher than those from biological equivalents of the same size, e.g., tadpole tails. By contrast, in MSMA samples with aspect ratio close to, or below, unity, uniaxial strains due to MFIS, rather than bending, is the dominant response [17] albeit with kinking if only one twin boundary carries the deformation [18, 19]. Chmielus et al. [20] reported large MFIS in polycrystalline Ni-Mn-Ga foam with single crystalline or bamboo struts. They discussed strut hinging (MTIB) and strut straining (MFIS) as possible mechanisms leading to large overall strain without conclusive statement regarding which mechanism dominates.

In this work, we study the effects of aspect ratio and field strength on MTIB and MFIS of Ni-Mn-Ga single crystal rods with small cross-sections $\left(1 \times 1 \mathrm{~mm}^{2}\right)$ and length of 4-12 $\mathrm{mm}$. Each sample was mounted to the sample holder such that the first $2 \mathrm{~mm}$ of the sample were rigidly embedded in a notch. The length of the freely movable portion of the beam ranged from 2 to $10 \mathrm{~mm}$ corresponding to aspect ratios ranging from 2 to 10 . The overall deformation state is a combination of axial strain and bending. Axial strain dominates only for short samples.

\section{Experimental Methods 2.1 Sample Preparation}

High purity nickel (99.999 wt\% purity, American Elements), manganese (99.999 wt\% and less than 110 ppm oxygen, Institute of Physics, Polish Academy of Sciences) and gallium (99.9999 wt\% purity, Atlantic Metal) were inductively melted under high vacuum to create an alloy of nominal composition $\mathrm{Ni}_{51.2} \mathrm{Mn}_{26.6} \mathrm{Ga}_{22.2}$ (numbers indicate atomic percent). One single crystal ingot was prepared from this alloy in a custom-built furnace using the Bridgman-Stockbarger technique. A complete description of the single 
crystal growth process using this custom furnace is described by Kellis [21]. The crystal was oriented using a Bruker D8 Discover x-ray diffractometer and then cut with a Princeton Scientific precision wire saw into a parallelepiped specimen with dimensions approximately $1 \times 1 \times 12 \mathrm{~mm}^{3}$ with all faces parallel to the $\{100\}$. All four long faces were polished by hand to 2000 grit ( $\sim 6 \mu \mathrm{m}$ surface finish) using silicon carbide paper.

The composition was measured along the beam specimen in a Hitachi-3400N-II analytical Scanning Electron Microscope (SEM) with an Energy-Dispersive X-ray spectrometer (EDX). The SEM was operated at a $20 \mathrm{kV}$ accelerating voltage, with a 10 mm working distance and a high purity nickel slug was used as the standard. The reported values were an average of 5 sites (composed of three point scans and three area scans) that were collected on one long face of the specimen near both ends and in the middle of the sample. The average composition of the beam specimen was $\mathrm{Ni}_{51.6 \pm 0.1}$ $\mathrm{Mn}_{25.1 \pm 0.2} \mathrm{Ga}_{23.3 \pm 0.2}$ (errors are the standard deviation of all measurements, in at.\%).

The crystal structure was determined using a Bruker AXS D8 Discover X-ray Diffractometer (XRD) with a $\mathrm{Cu}-\mathrm{K} \alpha$ source paired with a Hi-Star area detector. For phase analysis, the angles of the incident beam and of the detector center were simultaneously increased in increments of $5^{\circ}$ so that the $2 \theta$ angle covered the range from $60^{\circ}$ to $80^{\circ}$. A small piece (ca. $0.8 \times 1.1 \times 2.2 \mathrm{~mm}^{3}$ ), that was cut off from the sample after rotating field experiments, was placed on an acrylic sample holder and the sample stage translated and rotated such that the beam periodically covered the entire sample while diffracted intensity was collected for $300 \mathrm{~s}$. This procedure was repeated for a total of three sample faces. A pseudo-tetragonal (10M) structure with several twin variants was found while the calculated lattice parameters $(a, c)$ extracted from three faces of the beam 
specimen yielded $a=0.592 \mathrm{~nm}$ and $c=0.556 \mathrm{~nm}$ with a $c / a$ ratio of 0.94 which indicates a twinning stain $\varepsilon_{\mathrm{tw}}=1-c / a=6 \%$. The orientation of all surfaces deviated from $\{100\}$ by less than $5^{\circ}$.

The martensitic transformation temperatures were $M_{\mathrm{s}}=39^{\circ} \mathrm{C}, M_{\mathrm{f}}=37^{\circ} \mathrm{C}, A_{\mathrm{s}}=44^{\circ} \mathrm{C}, A_{\mathrm{f}}$ $=47^{\circ} \mathrm{C}$, which yields an equilibrium temperature $T_{\mathrm{M}}=42^{\circ} \mathrm{C}$ [22], and the Curie temperature $\left(T_{\mathrm{C}}\right)$ of the beams were determined using a MicroSense Model 10 Vibrating Sample Magnetometer (VSM). A small piece of the beam specimen was mounted with the long axis parallel to the static magnetic field of $19.9 \mathrm{kA} / \mathrm{m}[0.025 \mathrm{~T}]$ and magnetization was measured as a function of temperature with a heating rate of $5{ }^{\circ} \mathrm{C} / \mathrm{min}$ and a cooling rate of $5{ }^{\circ} \mathrm{C} / \mathrm{min}$.

The sample was fully martensite during experiments conducted at room temperature (20 $\pm 2{ }^{\circ} \mathrm{C}$ ) with a $\mathrm{T}_{\mathrm{M}}=42 \pm 1{ }^{\circ} \mathrm{C}$ and $\mathrm{T}_{\mathrm{C}}=91 \pm 1^{\circ} \mathrm{C}$. The martensitic phase is required for large bending deformation (MFIB) [14].

Magnetic-field-induced reorientation (MIR) [6] was determined with the VSM. A small portion of the beam specimen (ca. $0.8 \times 1.1 \times 2.2 \mathrm{~mm}^{3}$ ) was compressed along its longest axis, measured with a caliper and then embedded into a small piece of Styrofoam to allow twin movement but prevent sample rotation during the experiment. The sample was oriented with the longest axis parallel to the applied magnetic field and data was collected from 0 to $1194 \mathrm{kA} / \mathrm{m}[1.5 \mathrm{~T}]$ in $7.9 \mathrm{kA} / \mathrm{m}$ [0.01 T] steps with a dwell time of $1 \mathrm{~s}$. The magnet was then rotated such that the samples shortest axis was parallel to the applied magnetic field and data was collected from 0 to $716 \mathrm{kA} / \mathrm{m}[0.9 \mathrm{~T}]$ in $7.9 \mathrm{kA} / \mathrm{m}[0.01 \mathrm{~T}]$ steps with a dwell time of $1 \mathrm{~s}$. Finally the magnet was oriented parallel with the longest 
axis and data was collected from 0 to $716 \mathrm{kA} / \mathrm{m}[0.9 \mathrm{~T}]$ in $7.9 \mathrm{kA} / \mathrm{m}[0.01 \mathrm{~T}]$ steps with a dwell time of $1 \mathrm{~s}$.

\subsection{Rotating field experiments}

\subsubsection{Setup and Data Collection}

Rotating field experiments were performed with a custom built optical magnetomechanical device (OMMD) [23]. A rotatable PVC tube was inserted in an electromagnet such that the tube axis lay parallel between the surfaces of the pole pieces, i.e., perpendicular to the magnetic field direction. At one end of the tube, the beam specimen was mounted perpendicularly to the tube axis in the following manner. The beam was glued at one end into a small trench within a brass block, leaving the other end of the beam free. The brass block was rigidly attached to the plastic sample holder using brass screws. The sample holder assembly was then slid into the PVC tube right next to an illumination assembly. A camera was rigidly mounted to the opposite end of the tube so that the beam was in the field of view of the camera. Both the tube and camera were attached to a gear and motor assembly, on the OMMD, and were rotated with a stepper motor at $1^{\circ}$ increments.

While the tube, sample and camera rotated counter-clockwise around the tube axis (Fig. 1), the camera recorded the beam, seemingly at rest in a clockwise rotating field, as shown in Fig. 2(a). The field direction, measured by the angle $\alpha$ as defined in Fig. 2(a), changed gradually from parallel $\left(\alpha=0^{\circ}\right)$ to perpendicular to the beam axis $\left(\alpha=90^{\circ}\right)$, back to parallel $\left(\alpha=180^{\circ}\right)$ and again to perpendicular $\left(\alpha=270^{\circ}\right)$ and finally to parallel $\left(\alpha=360^{\circ}\right)$. For the remainder of this text, we use the camera coordinate system that 
rotates with respect to the laboratory coordinate system. Thus, the field direction rotates around the sample. Within the OMMD, the stepper motor and a rotary encoder, with 2500 pulses per revolution, controlled the rotary which yielded a resolution of $\Delta \alpha= \pm 0.1^{\circ}$ in rotational movement. The data were collected onto the computer using LabVIEW to interface with the camera and the microcontroller.

At room temperature, $20 \pm 2^{\circ} \mathrm{C}$, a magnetic field of $79.6 \mathrm{kA} / \mathrm{m}[0.1 \mathrm{~T}]$ was first applied parallel to the long beam axis. The field was then rotated clockwise and 361 images of the beam were taken in $1^{\circ}$ increments from 0 to $360^{\circ}$. The field was then rotated counterclockwise from 360 to $0^{\circ}$ in $1^{\circ}$ increments. The magnetic field was then increased to $159 \mathrm{kA} / \mathrm{m}[0.2 \mathrm{~T}]$ and rotated using the same parameters described above. Tests were repeated with the following applied fields: from $79.6[0.1 \mathrm{~T}]$ to $796 \mathrm{kA} / \mathrm{m}[1.0 \mathrm{~T}]$ in 79.6 $\mathrm{kA} / \mathrm{m}[0.1 \mathrm{~T}]$ increments, followed by 955 [1.2], 1114 [1.4], and 1353 [1.7] kA/m [T]. Then the brass block was unscrewed from the sample holder and then the sample was cut with a wire saw to reduce its length by approximately $2 \mathrm{~mm}$ without removing the sample from the brass block. The brass block was then reattached to the plastic sample holder and the series of experiments were repeated. Six series of experiments were performed with free sample length $l$, defined in Fig. 2(b), of 10, 8, 6, 4, 3, and 2 mm.

\subsubsection{Image Analysis}

The collected data were in the form of approximately 35,000 images with the associated camera angle of rotation. The images were analyzed using a custom-written Matlab code. The images were first aligned by using a fiducial mark placed near the sample in the sample chamber as a reference. For each image, the top and bottom edge of the sample 
were found at about 125 points (Fig. 2(b)). The edge line was approximated by

interpolating the edge points using a quadratic function, as shown in Fig. 2(c), showing the points found at the top of the picture and the parabolic interpolation without the picture. The $R^{2}$ value for the interpolation of the top edge of the sample consistently exceeded 0.9 .

Using the quadratic model for the deformed sample, the curvature $(k)$ as a function of position on the sample is:

$$
k=\frac{\frac{d^{2} y}{d x^{2}}}{\left(1+\left(\frac{d y}{d x}\right)^{2}\right)^{\frac{3}{2}}}
$$

where $y$ is the vertical coordinate and $x$ is the horizontal coordinate in pixels. The free end of the sample was found using edge detection. The bisecting line of the sample, found by averaging the polynomial models of the top and bottom of the sample, was used to find the length of the sample in each image. The length $(l)$ was determined with the arc length equation:

$$
l=\int_{a}^{b} \sqrt{1+\left(\frac{d y}{d x}\right)^{2}} d x
$$

where $a$ and $b$ are the endpoints of the line segment.

Bending strain $\left(\varepsilon_{\mathrm{B}}\right)$ was found using the curvature

$$
\varepsilon_{\mathrm{B}}=\frac{d * k}{2}
$$

where $d$ is the width of the sample. This yields a positive value when the beam bends upwards as shown in Fig. 2(b). Thus, $\varepsilon_{\mathrm{B}}$ is the normal component of the strain tensor at the lower edge of the beam (i.e. at the blue line in Fig. 2(b)) parallel to the beam axis at the root of the beam (marked by white dot in Fig. 2(b)). The magnitude of $\varepsilon_{\mathrm{B}}$ is greatest near the point of fixation. The quadratic equation used to approximate the bending 
deformation is a simple model that accounts for the decrease in $\varepsilon_{\mathrm{B}}$ as the distance from the fixture increases. The fit deviates in such a way that curvature and bending strain are underestimated near the fixed end.

Axial strain $\left(\varepsilon_{\mathrm{A}}\right)$ was found using the length of the sample:

$$
\varepsilon_{\mathrm{A}}=\frac{l-l_{0}}{l_{0}}
$$

where $l_{0}$ is the minimum length and $l$ is the length at a given field angle.

Axial strain $\left(\varepsilon_{\mathrm{A}}\right)$ and bending strain $\left(\varepsilon_{\mathrm{B}}\right)$ contribute to the same normal component of the strain tensor along the beam length axis. Thus, the sum of $\varepsilon_{\mathrm{A}}$ and $\varepsilon_{\mathrm{B}}$ is the total normal strain along the beam length at the lower edge of the beam (i.e. at the left end of the blue line in Fig. 2(b)).

\subsection{Mechanical Testing and Magnetization Curves}

After performing the rotating field experiment, a portion of the beam (ca. $0.8 \times 1.1 \times 2.2$

$\mathrm{mm}^{3}$ ), that was cut with a wire saw between rotating field test series, was tested in a compressive mechanical test bench (Zwick 1445) equipped with a $500 \mathrm{~N}$ load cell (MTS) and optical extensometers (Heidenhain D-83301, Traunreut) with resolutions of $0.5 \mathrm{~N}$ in load and $10 \mathrm{~nm}$ in displacement [24]. Because of the small size of the sample, the cross section has a large relative error. Additional errors in the stress measurements may originate from non-parallelism of the cut faces and from friction. Thus, the stress values have a large systematic error though the statistical error is small. The sample was first placed in the VSM with its shortest dimension $(0.8 \mathrm{~mm})$ parallel to a $796 \mathrm{kA} / \mathrm{m}$ [1.0 T] applied magnetic field for approximately $60 \mathrm{~s}$ to elongate the sample in the long axis. The sample dimensions were recorded with a caliper. The sample was then placed into a 
compression cage, with its longest axis $(2.2 \mathrm{~mm})$ parallel to the compression direction. The displacement of the sample was recorded as the sample was loaded in compression from 0 to $8.06 \mathrm{~N}$ and then unloaded to $0.24 \mathrm{~N}$, with a constant crosshead speed of 1.25 $\mu \mathrm{m} / \mathrm{s}$.

After performing the compression tests, three magnetization curves were measured on the same sample. In the first experiment, the magnetic field was applied parallel to the samples longest $(2.2 \mathrm{~mm})$ direction. The magnetic field was increased from 0 to 1194 $\mathrm{kA} / \mathrm{m}[1.5 \mathrm{~T}]$ and reduced back to zero. Then the magnetic field direction was changed to align with the shortest $(0.8 \mathrm{~mm})$ direction and the magnetization experiment was increased from 0 to $716 \mathrm{kA} / \mathrm{m}[0.9 \mathrm{~T}]$. Finally, the magnetic field direction was rotated back and the magnetization experiment was performed again with the field parallel to the longest sample direction with the field increasing to $716 \mathrm{kA} / \mathrm{m}[0.9 \mathrm{~T}]$. The magnetic field ramp rates were $0.5(\mathrm{kA} / \mathrm{m}) / \mathrm{s}$.

\section{Results}

\subsection{Magnetization and uniaxial compressive deformation}

Fig. 3(a) shows magnetization curves measured on a beam with size $2.2 \times 1 \times 0.8 \mathrm{~mm}$. When the field was applied parallel to the long axis (green-gray dashed lines), the magnetization curve had a steep slope without noticeable positive curvature. Upon removal of the magnetic field the sample demagnetized without noticeable hysteresis. When the magnetic field was applied parallel to the shortest axis $(0.8 \mathrm{~mm})$, the slope of the magnetization curve was smaller than for the field perpendicular to this direction. The slope increased, between $350[\sim 0.4]$ and $450 \mathrm{kA} / \mathrm{m}[\sim 0.5 \mathrm{~T}]$, until close to saturation at $475 \mathrm{kA} / \mathrm{m}[\sim 0.6 \mathrm{~T}]$ (bottom curve). Upon removal of the magnetic field, the sample 
demagnetized with a significant hysteresis. Yet, the slope at low magnetic fields was still flatter than for magnetization along the longest sample direction. This is consistent with the large demagnetization factor when magnetized along the short direction.

The stress-strain curve for a $2.2 \mathrm{~mm}$ long sample of the beam specimen, shown in Fig. 4(b), displayed a maximum strain of $6 \%$ at $8 \mathrm{MPa}$. Upon loading, the sample started deforming plastically at a stress of $\sim 1 \mathrm{MPa}$. The stress increased monotonically with increasing deformation. Upon unloading, the sample first dropped rapidly, and then more slowly, while the strain recovered to a final value of $\sim 4 \%$.

\subsection{Magnetic field induced deformation}

Fig. 4(a) shows the bending strain $\left(\varepsilon_{\mathrm{B}}\right)$ as a function of position for an $8 \mathrm{~mm}$ beam subjected to a $1353 \mathrm{kA} / \mathrm{m}[1.7 \mathrm{~T}]$ magnetic field. The maximum curvature, and thus the maximum bending strain, occurs at the fixed end, i.e. at $x=0$. This curvature value was plotted against the field angle in Fig. 4(b) for clockwise (solid) and counter-clockwise (dashed) rotation. Starting at $\alpha=0^{\circ}$, the beam bent downward with increasing field angle. At about $70^{\circ}$, the bending strain reached a local minimum and reversed at about $90^{\circ}$. Upon further increasing the angle, the bending strain reached a local maximum at about $110^{\circ}$ and decreased towards zero at about $180^{\circ}$. Between 180 and $360^{\circ}$, the bending strain repeated this behavior. The forward (clockwise) and backward (counterclockwise) bending strains exhibited a hysteresis of about $12 \pm 3^{\circ}$.

Both axial and bending strains were present for all lengths of the sample at an applied magnetic field of $796 \mathrm{kA} / \mathrm{m}[1.0 \mathrm{~T}]$, as shown in Fig. 5(a, b) where only the clockwise rotation is displayed for clarity. The data for the $2 \mathrm{~mm}$ long sample, shown in Fig. 5(a), was shifted up to zero strain because the beam was angled down slightly after cutting and 
the exerted bending strain was not enough to straighten out the beam. The axial strain exhibited two sharp maxima at 90 and $270^{\circ}$ and two broad minima at $0 / 360^{\circ}$ and $180^{\circ}$. The maximum axial strain $\left(\varepsilon_{\mathrm{A}}\right)$ was consistent at $\sim 1.7 \%$ for all sample lengths while the maximum bending strain $\left(\varepsilon_{\mathrm{B}}\right)$ increased with increasing sample length. Axial strain dominated deformation for beams shorter than $8 \mathrm{~mm}$ and bending strain was dominant for the two longest samples, as shown in Fig. 5(c). Similarly, as the applied magnetic field increased for one length of the beam, the bending strain increased and the axial strain remained constant with the exception of the lowest value of $159 \mathrm{kA} / \mathrm{m}[0.2 \mathrm{~T}]$ as shown in Fig. $6(\mathrm{a}, \mathrm{b})$ on the $8 \mathrm{~mm}$ long sample. In Fig. 6(c) the maximum slope of $\mathrm{d} \varepsilon_{\mathrm{B}} / \mathrm{d} \alpha$, which occurred between the angles $90^{\circ}$ and $100^{\circ}$, was plotted as a function of the applied magnetic field.

\section{Discussion}

\subsection{Uniaxial compressive deformation}

The compressive stress-strain curve, Fig. 3(b), revealed onset of plastic deformation at a very low stress of $\sim 1 \mathrm{MPa}$, and twinning deformation close to $6 \%$ strain (stress of $7 \mathrm{MPa}$ ) above which a sharp increase in slope indicates twinning exhaustion (i.e. onset of further elastic deformation). The magneto-stress for $10 \mathrm{M} \mathrm{Ni-Mn-Ga}$ is reported to be about 2-3 MPa [18]. This stress level is reached at a strain of about $1.5 \%$ in Fig. 3(b) that corresponds well with the axial strain of $1.7 \%$ measured in most of the rotating field experiments in Fig. 5(c). Once the magneto-stress is surpassed by an applied magnetic field, the axial strain remains consistent as shown in Fig. 6(b), which implies that beyond the magneto-stress required to move twin boundaries there is no applied field dependence. Thus, axial strain increases with decreasing twinning stress. The bending 
strain is a function of torque which is in first approximation proportional to the length of the beam. The slope of the curve for bending strain in Fig. 5(c) would probably increase with decreasing twinning stress. However, bending strain would remain small for short samples. Thus, it is possible that for a beam sample with lower twinning stress, the relative importance of axial and bending strain in rotating field would be shifted towards more axial strain as the sample aspect ratio decreases.

In Fig. 3(b), the unloading part of the stress-strain curve exhibits a large recovery of nearly $4 \%$ when the stress falls below about $5 \mathrm{MPa}$. Chmielus et al. [19] reported that, for a monocrystalline $14 \mathrm{M}$ sample with $2.4 \times 3.0$ x $4.4 \mathrm{~mm}$ dimensions, large compressive unloading strain recovery of $\sim 1 \%$ could be attributed to the relatively rough surface of a sample polished with $6 \mu \mathrm{m}$ diamond slurry. A deformed surface layer impedes twin boundary motion near the surface. During mechanical loading, surface pinning of twin boundaries leads to pile-ups of twinning dislocations, which act as a restorative force when the applied stress is removed.

\subsection{Magnetic field induced deformation}

The large recovery strain upon uniaxial compressive unloading implies that without magnetic field or mechanical load, the orientation of the short axis of the unit cell $c$ in the monocrystalline beam is perpendicular to the beam axis since the sample is expanded and, thus has its $a$-axis is parallel to the beam length direction. This explains why only little twinning is detected in the magnetization curve with the field applied parallel to the short beam axis (Fig. 3(a)). Twinning gives rise to a positive curvature of the magnetization curve between $350[\sim 0.4]$ and $450 \mathrm{kA} / \mathrm{m}[\sim 0.5 \mathrm{~T}]$. This change is subtle and indicates that twinning is sluggish, consistent with the relatively steep slope of the 
deformation curve (Fig. 3(b)). Such a sluggish behavior differs from the fast switching occurring in softer Ni-Mn-Ga with a stress plateau at stress levels below $1 \mathrm{MPa}$ for long and short single crystals $[6,18,21]$.

The bending strain and axial strain were extracted from images collected with the OMMD. Bending and axial strains are zero where the applied magnetic field is parallel to the sample $\left(0 / 360^{\circ}\right.$ and $\left.180^{\circ}\right)$. At these specific angles and with sufficiently strong magnetic fields, the crystallographic $c$-axis (short axis of the unit cell) is parallel to the magnetic field so that the sample is short and straight. With slowly increasing field angle and at field strength below the saturation field, the magnetic anisotropy energy forces the magnetic moments to stay aligned with the crystallographic $c$-axis. The resulting Zeeman energy provides a torque, which bends the sample in the direction of the magnetic field. The twinning arrangements resulting in bending and axial strain are schematically illustrated in Fig. 7 where twins with $c$ parallel and perpendicular to the long beam axis are colored blue and orange, respectively. An equal distribution of blue and orange twin domains with parallel twin boundaries, results in an axial strain of $(1-c / a) / 2=3 \%$, Fig. 7(a). An increase in the fraction of orange twins with the long $a$ parallel to the beam axis, Fig. 7(b), results in an increase in axial strain. A twin pattern with triangular twins of equal twin fraction result in $\pm 3 \%$ bending strain as displayed in Fig. 7(c). Fig. 7(d) displays a twin microstructure with a combination of axial and bending strain where the triangular shape of the blue twins causes bending and the larger fraction of orange twins causes an axial strain larger than $3 \%$.

For field angles $(\alpha)$ of $\sim 0 / 360$ and $180^{\circ}$, bending strain changes sign from positive to 
negative where the slope is relatively flat. For field angles $(\alpha)$ of $\sim 90$ and $270^{\circ}$, the bending strain direction changes with a strong slope and a peak in axial strain of about $1.7 \%$ appears. This $1.7 \%$ strain corresponds to about one quarter of the theoretical limit of $6 \%$ axial strain, which would occur when $c$ is perpendicular to the beam axis in the entire sample. Thus, in one quarter of the beam volume, the crystallographic $c$-axis is perpendicular to the beam axis (hence parallel to the current magnetic field) and in the rest of the sample volume, the $c$-axis is parallel to the beam axis (hence perpendicular to the magnetic field). The smooth curvature of the beam implies that many twin boundaries are present because an isolated twin boundary would cause a distinct kink $[15,16]$. At field angles slightly below and slightly above $90^{\circ}$, the torque is strong and causes bending strain which competes with axial strain. At one side of the sample, the bending strain is tensile as is the uniaxial MFIS strain, and thus both strains are additive. At the other side, the bending strain is compressive and subtracts from the tensile uniaxial MFIS strain. The strong torque around 90 and $270^{\circ}$ produces bending and, thus, reduces axial strain. As a result, axial strain exists only in a narrow angle range as evidenced by sharp peaks shown in Fig. 5(b) and 6(b). The weak torque around $0 / 360^{\circ}$ and $180^{\circ}$ does not compete with MFIS which leads to broad minima of axial strain at these angles. This asymmetry of maxima and minima is in contrast to single crystal samples with an aspect ratio smaller than 2, which have symmetrical broad maxima and minima of MFIS [25].

For magnetic field angles in the $75-120^{\circ}$ and the $250-295^{\circ}$ ranges, a rapid change in bending occurs in Fig. 5(a) and 6(a), as measured by the maximum value of $\mathrm{d} \varepsilon_{\mathrm{B}} / \mathrm{d} \alpha$. Fig. 6(c) shows that the maximum rate $\left(\mathrm{d} \varepsilon_{\mathrm{B}} / \mathrm{d} \alpha\right)_{\max }$ increases until $637 \mathrm{kA} / \mathrm{m}[0.8 \mathrm{~T}]$ and then 
wanes. This change is not simply due to the increase of bending strain. The magnetic saturation of $62 \mathrm{emu} / \mathrm{g}$ is achieved near the saturation field of about $640 \mathrm{kA} / \mathrm{m}[\sim 0.8 \mathrm{~T}]$ for $10 \mathrm{M} \mathrm{Ni}-\mathrm{Mn}-\mathrm{Ga}$ [26]. There are three forces acting on the material with origin in shape anisotropy energy, magnetic anisotropy energy, and Zeeman energy. For small magnetic fields, all three forces favor bending. With increasing magnetic field, the Zeeman energy (being proportional to the field) overcomes the magnetic anisotropy energy (which saturates at large fields) and magnetic moments eventually align with the magnetic field direction independent of orientation of the field and of the crystallographic $c$ direction [18]. At this point, only the shape anisotropy contributes to bending. The reduction in $\left(\mathrm{d} \varepsilon_{\mathrm{B}} / \mathrm{d} \alpha\right)_{\max }$ with increasing magnetic field implies a reduction of the net magnetic-field-induced torque. We speculate that the change in relative contributions of the driving forces for the bending torque is responsible for the changing dynamics at the reversal of bending around 90 and $270^{\circ}$. A detailed micromagnetic analysis is necessary to substantiate this hypothesis, which is outside the scope of this study.

\subsection{Engineering Applications}

Bending deformation is not desired in linear actuators such as those proposed by Schlüter et al. [3]. In such actuators, bending leads to friction against the guiding walls, wear, and fatigue and, thus, reduced actuator performance. In contrast, biological structures utilize bending mechanisms in various ways. Flagella and cilia propel microorganisms in water and fish use their tails and fins for the same purpose. Birds, bats and insects produce lift through flapping their wings. Flowers open and close their corolla by bending their petals and turn their corolla by bending their stems. Flaps bend to control the flow of blood in 
the heart. Similarly, bending actuators based on MTIB may propel small objects through fluids as proposed by Ganor et al. [15] and Kanner et al. [16]. Such a device could also actuate small mechanisms (e.g. position mirrors), or serve as valves in fluidic systems. The design of such an actuator is very simple: an MSMA beam and a set of electromagnetic coils. This simplicity and the fully solid-state design (i.e., no hydraulics or pneumatics) without moving parts (i.e., without gears or hinges) enables simple miniaturization of various devices, including mobile microrobots, microfluidic apparatuses and micro-surgical instruments.

\section{Conclusions}

The deformation of a small $10 \mathrm{M} \mathrm{Ni}_{51.6} \mathrm{Mn}_{25.1} \mathrm{Ga}_{23.3}$ monocrystalline beam in a rotating magnetic field was studied at several applied magnetic fields $(79.6-1353 \mathrm{kA} / \mathrm{m}[0.1-1.7$ T]) and sample aspect ratios (2-10). As the field rotates, the beam deforms uniaxially via magnetic field induced straining (MFIS) as well as by bending via magnetic torque induced bending (MTIB). The results show the following trends:

- With increasing beam aspect ratio, the maximum bending strain range increases from 0.4 to $2.3 \%$ (as determined from curvature measurements). This is because the torque induced on a beam by a magnetic field increases with its length. By contrast, with increasing beam aspect ratio, the axial strain is constant at about $1.7 \%$, as expected since the magneto-stress is independent on sample shape.

- With increasing magnetic field magnitude, the bending strain range increases from 0.33 to $1.89 \%$ because the contribution of the Zeeman energy to the magnetic torque is proportional to the magnetic field. Again, by contrast, as the magnetic field 
increases, the axial strain is constant at $\sim 1.7 \%$, except for the lowest field. This is because the magneto-stress saturates at the large magnetic fields used here.

Bending from magnetic torque - a deformation mode active in all ferromagnetic samples with a high aspect ratio (such as wires, rods or beams) - is accommodated by twinning deformation in MSMA beams with very low twinning stresses (such as martensitic Ni$\mathrm{Mn}-\mathrm{Ga}$ ), thus resulting in large bending deflection. This is unlike ferromagnetic samples with high twinning or yield stress (such as Fe-Mn-Si, Fe-Ni-Co-Ti, and Fe), which remain elastic and thus show only small deflections. In MSMA actuators, this bending effect is undesirable if actuation is only allowed in one direction (as it creates friction); it can however be desirable if deformation of the actuator is expected to follow the magnetic field freely in space in all directions, thus allowing biomimetic design solutions inspired by biological flagella, cilia, valves, leaves, stems, tails, and wings.

\section{Acknowledgments}

The US National Science Foundation supported this research at Boise State University through Grant No. DMR-1207192 (NJK, CLP, and PM) and Grant No. DMR-MRI 0619795 (x-ray diffractometer), and at Northwestern University through Grant No. DMR-1207282 (DCD).

\section{References}

[1] Kohl M, Brugger D, Ohtsuka M, Takagi T. Sensors Actuators A Phys 2004;114:445.

[2] Kohl M, Brugger D, Ohtsuka M, Krevet B. Sensors Actuators A Phys 2007;135:92. 
[3] Schlüter K, Holz B, Raatz A. Adv Eng Mater 2012;14:682.

[4] Le Gall Y, Bolzmacher C. Microsyst Technol 2013;20:533.

[5] Minorowicz B, Nowak A, Stefański F. J Achiev Mater Manuf Eng 2013;61:216.

[6] Heczko O. Mater Sci Technol 2014;30:1559.

[7] Ullakko K, Huang JK, Kantner C, Handley RCO, Kokorin V V. Appl Phys Lett 1996;69:1966.

[8] Straka L, Hänninen H, Lanska N, Sozinov A. J Appl Phys 2011;109:063504.

[9] Smith A, Tellinen J, Müllner P, Ullakko K. Scr Mater 2014;77:68.

[10] Schiepp T, Detkov V, Maier M, Pagounis E, Laufenberg M. Failure Mechanisms and High-Cycle Fatigue of MSM Acuators, in:. ICFSMA' 13 Fourth Int. Conf. Ferromagn. Shape Mem. Alloy. 2013.

[11] Schiepp T, Maier M, Pagounis E, Schlüter A, Laufenberg M. IEEE Trans Magn 2014;50:7024504.

[12] Kiang J, Tong L. J Magn Magn Mater 2007;313:214.

[13] Chen X, Moumni Z, He Y, Zhang W. J Mech Phys Solids 2014;64:249.

[14] Zheng P, Kucza NJ, Patrick CL, Müllner P, Dunand DC. J Alloys Compd 2015;624:226.

[15] Ganor Y, Shilo D, Zarrouati N, James RD. Sensors Actuators, A Phys 2009;150:277.

[16] Kanner OY, Shilo D, Sheng J, James RD, Ganor Y. Smart Mater Struct 2013;22:085030.

[17] Heczko O, Sozinov a., Ullakko K. IEEE Trans Magn 2000;36:3266.

[18] O’Handley RC, Murray SJ, Marioni M, Nembach H, Allen SM. J Appl Phys 2000;87:4712. 
[19] Chmielus M, Witherspoon C, Ullakko K, Müllner P, Schneider R. Acta Mater 2011;59:2948.

[20] Chmielus M, Zhang XX, Witherspoon C, Dunand DC, Müllner P. Nat Mater 2009;8:863.

[21] Kellis D, Smith A, Ullakko K, Müllner P. J Cryst Growth 2012;359:64.

[22] Tong HC, Wayman CM. Acta Metall 1974;22:887.

[23] Rothenbühler A, Barney Smith EH, Müllner P. Application of Image Processing to Track Twin Boundary Motion in Magnetic Shape Memory Alloys, in:. Bingham PR, Lam EY (Eds.). SPIEIS\&T, vol. 8300. 2012.

[24] Müllner P, Chernenko VA, Kostorz G. Scr Mater 2003;49:129.

[25] Müllner P, Chernenko VA, Kostorz G. J Appl Phys 2004;95:1531.

[26] Jin X, Marioni M, Bono D, Allen SM, O’Handley RC, Hsu TY. J Appl Phys 2002;91:8222. 


\section{Figure Captions}

Fig. 1: Ni-Mn-Ga beam orientation for the counter-clockwise measurements in a magnetic field at four separate imaging conditions (a) 0 degrees, (b) 90 degrees, (c) 180 degrees, and (d) 270 degrees.

Fig. 2: (a) Camera coordinates of sample rotation for clockwise measurements in a magnetic field at four separate imaging conditions for $\alpha=0,90,180$, and $270^{\circ}$. (b) The red and blue lines on top and bottom, of an $8 \mathrm{~mm} \mathrm{Ni-Mn-Ga}$ beam at $\alpha=111^{\circ}$ with an applied magnetic field of $1,353 \mathrm{kA} / \mathrm{m}$ [1.7 T], represent 123 points on the sample edges spaced 5 pixels apart. The bisecting dashed line was found by averaging the two interpolated lines. (c) Plot of the position of the top edge of the beam (red line in $b$ ), along the length $(l)$ of the beam.. The red points are experimental values of deflection along length $(l)$ and the black solid line is the parabolic interpolation. The bisecting dashed line in (b) was found by averaging the two interpolated lines for the top and bottom edge with the origin shown as a white dot in Fig. 2(b). The fiducial marker (marked in b), seen just above the sample, was used for image alignment.

Fig. 3: (a) Plot of magnetization vs. magnetic field for $2.2 \mathrm{~mm}$ long sample. The switching field test displays a constant steep slope when the field is applied parallel to the long $(2.2 \mathrm{~mm}$ ) axis of the beam (green and purple-dashed curves) that indicates no twin boundary movement. When the magnetic field is applied parallel to the beam short axis (black curve) the slope increases, around $350 \mathrm{kA} / \mathrm{m}(\sim 0.4 \mathrm{~T})$, which indicated twinning (arrow). (b) Uniaxial compressive stress-strain curve for a $2.2 \mathrm{~mm}$ long portion of the beam sample, showing a maximum of $6 \%$ strain and $\sim 4 \%$ recovery along the longest axis of the sample.

Fig. 4: (a) The bending strain as a function of position from the brass holder for the $8 \mathrm{~mm}$ long sample for a magnetic field of $1353 \mathrm{kA} / \mathrm{m}[1.7 \mathrm{~T}]$ and angle $\alpha$ of $111^{\circ}$. (b) Plot of bending strain as a function of field angle for an $8 \mathrm{~mm}$ sample at $1353 \mathrm{kA} / \mathrm{m}$ [1.7 T], displaying a small hysteresis between clockwise rotation (solid line) and counterclockwise rotation (dashed line).

Fig. 5: (a) Bending strain as a function of magnetic field angle for various beam lengths for a $796 \mathrm{kA} / \mathrm{m}[1.0 \mathrm{~T}]$ applied magnetic field. (b) Corresponding axial strain for samples with various lengths at an applied field of $796 \mathrm{kA} / \mathrm{m} \mathrm{[1.0} \mathrm{T].}$ (c) Strain range (strain max - strain min) of bending strain as a function of sample length.

Fig. 6: (a) Bending strain as a function of magnetic field angle for an $8 \mathrm{~mm}$ sample at various applied magnetic fields. (b) Corresponding axial strain for the $8 \mathrm{~mm}$ sample at various applied magnetic fields. (c) Plot of maximum of the slope of $d \varepsilon_{B} / d \alpha$ as a function of magnetic field of an $8 \mathrm{~mm}$ sample exhibits a threshold for applied magnetic field at 796 $k A / m[1.0 T]$.

Fig. 7: Schematic, idealized twin patterns illustrating generation of axial strain and bending strain; blue and orange domains have c parallel and perpendicular to the long beam axis, respectively. (a) Equal fractions of blue and orange twins display a 3\% axial strain without bending; (b) Axial strain increases with increasing fraction of orange twins; (c) Triangular twins induce bending. (d) Small triangular blue twins in a majority of orange twins result in a combination of bending and axial straining. 
Figures

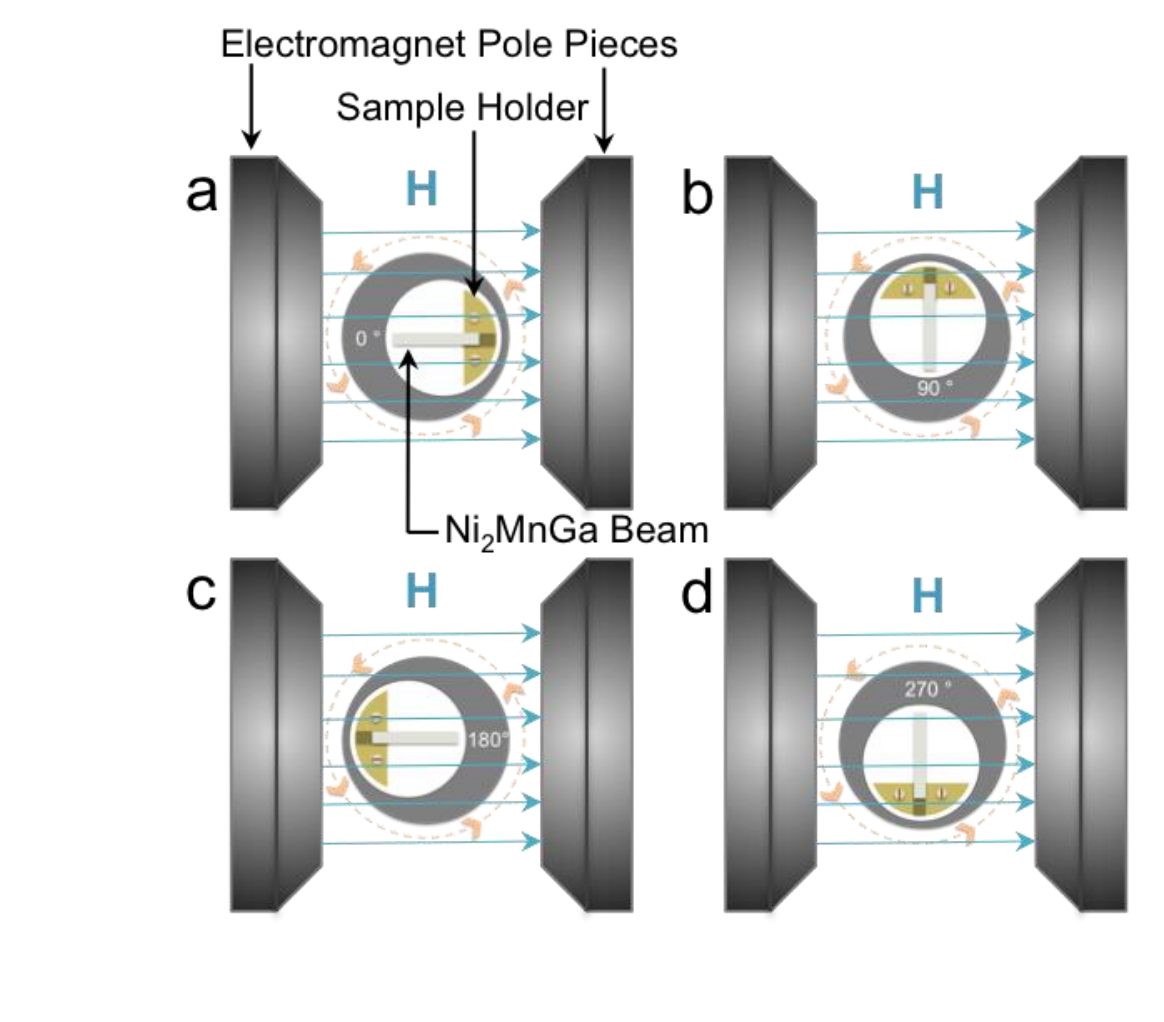

Figure 1

Figure(s)

Figure 1
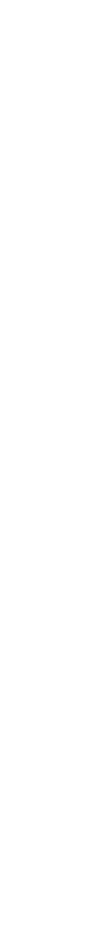

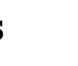

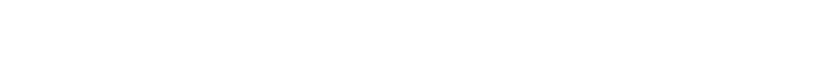

(n)

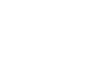

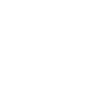

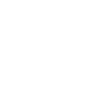$$
\text { (1) }
$$

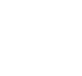
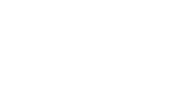

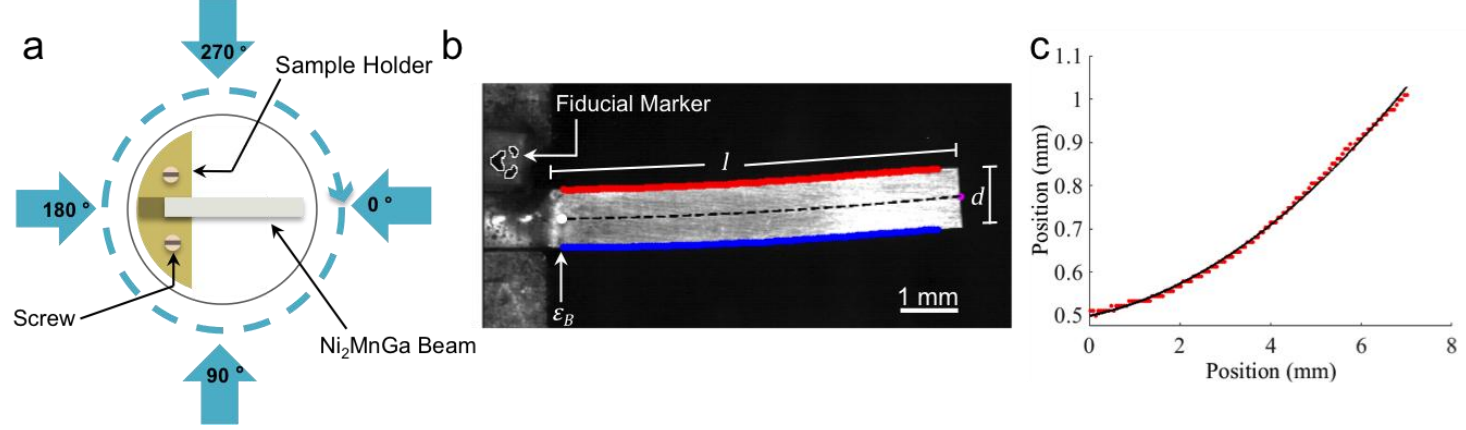

Figure 2 

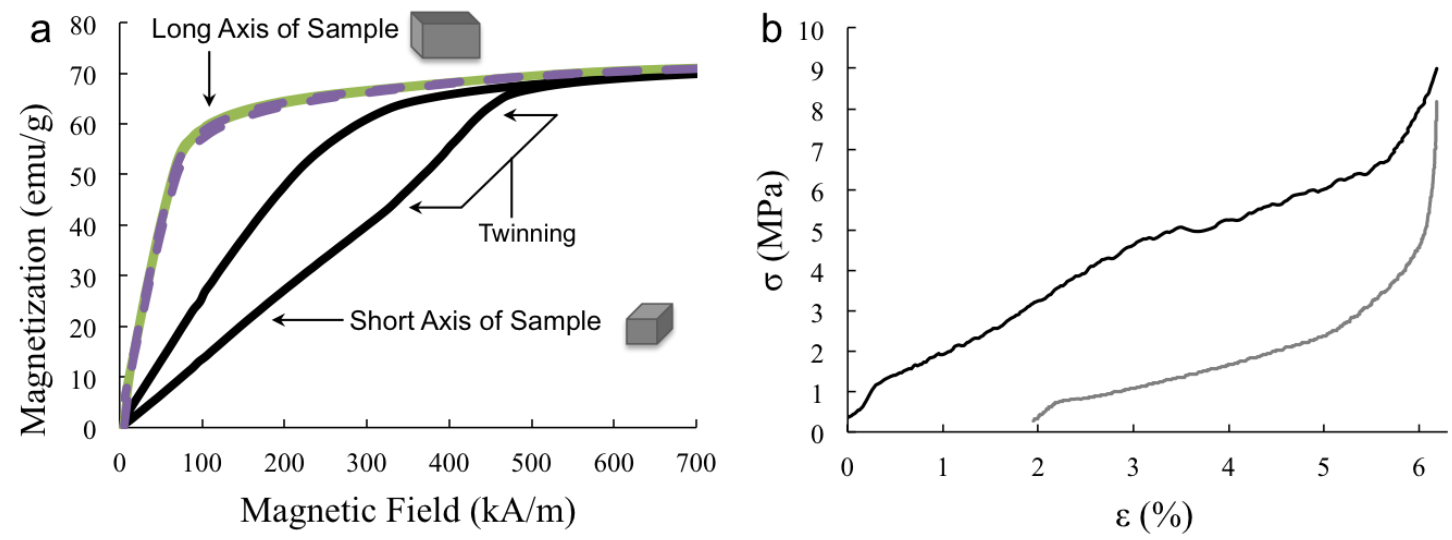

Figure 3 

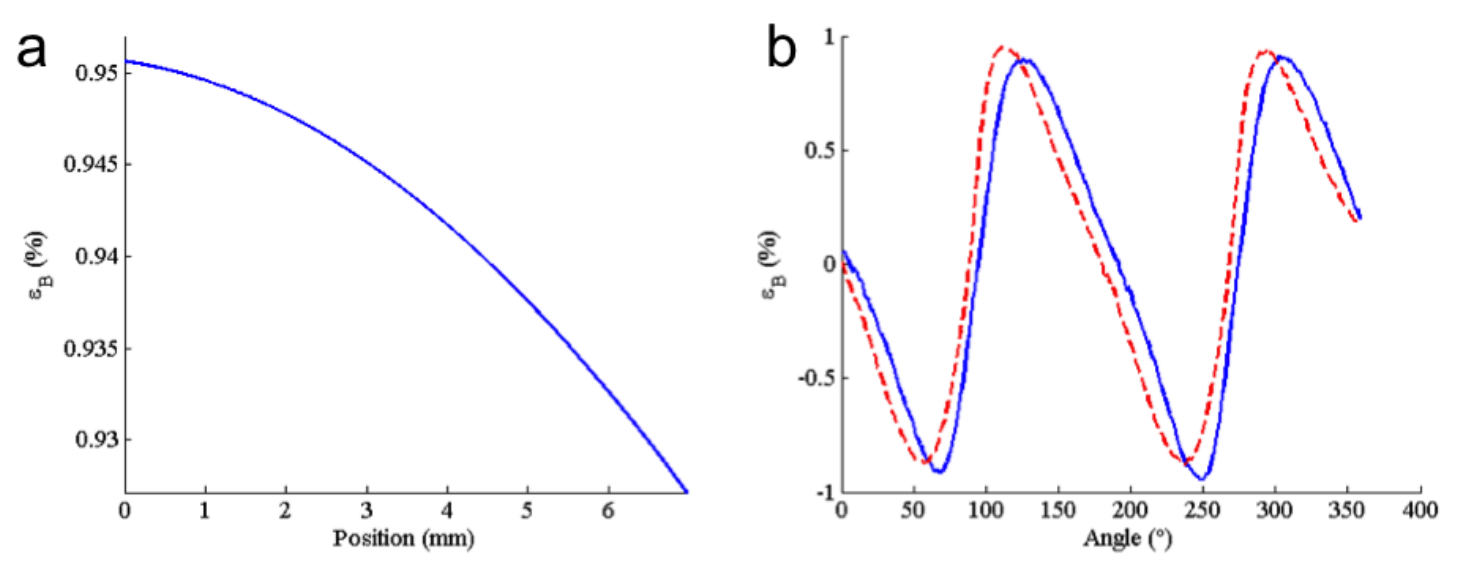

Figure 4 

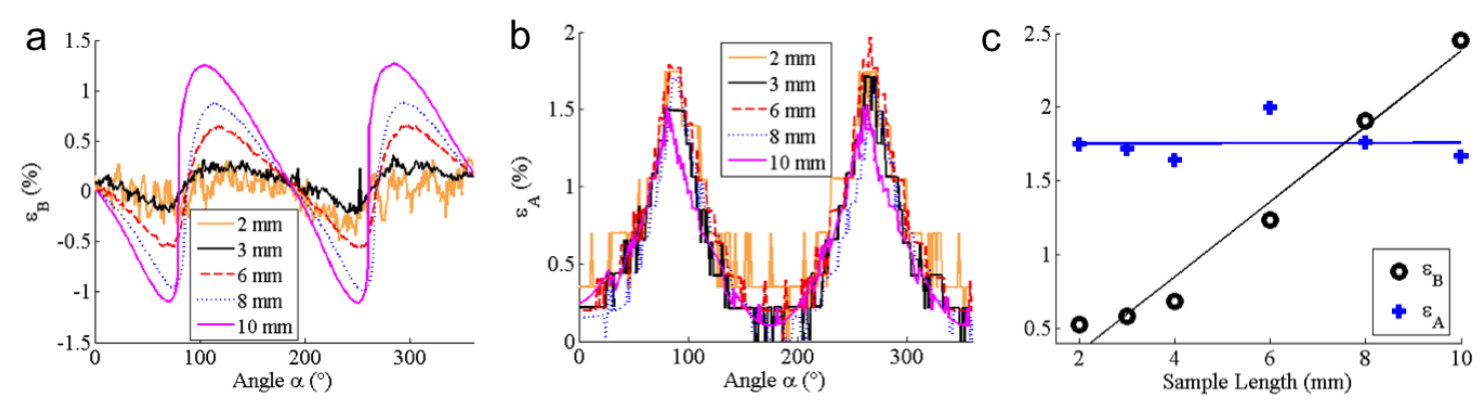

Figure 5 

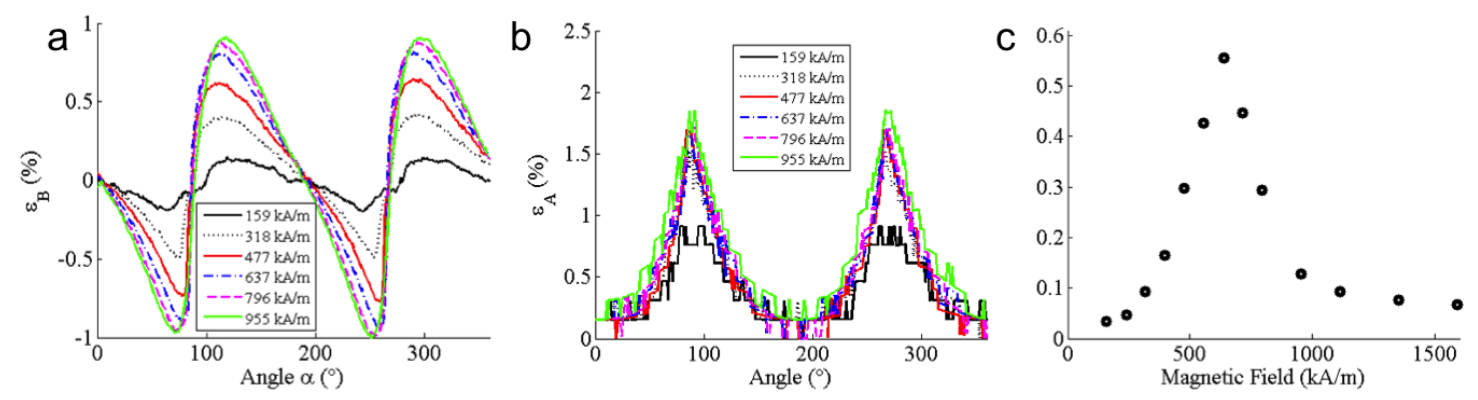

Figure 6 
(a)

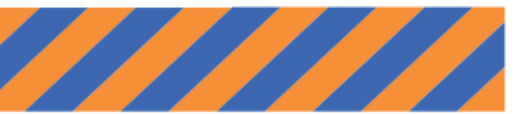

$c \square_{c}^{a}$

(b)

a

(c)

(d)

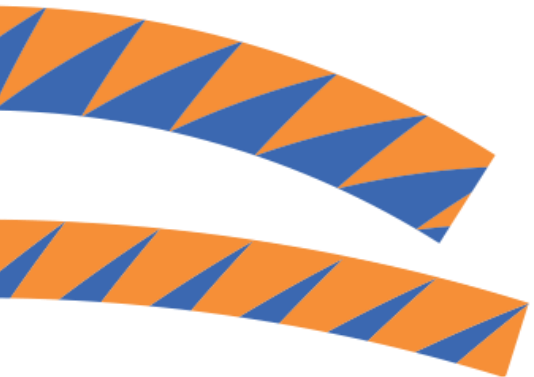

Figure 7 